\title{
Electric Power Generation Using Buoyancy-Induced Vortices
}

\author{
Mark W. Simpson ${ }^{1}$, Arne J. Pearlstein ${ }^{2}$ and Ari Glezer ${ }^{1}$ \\ ${ }^{1}$ Woodruff School of Mechanical Engineering \\ Georgia Institute of Technology \\ 771 Ferst Drive, Atlanta, GA 30308 \\ Phone/Fax number: 404-894-3266, e-mail: ari.glezer@me.gatech.edu \\ ${ }^{2}$ Department of Mechanical Science and Engineering \\ University of Illinois at Urbana-Champaign \\ 1206 West Green Street, Urbana, IL 61801
}

\begin{abstract}
Thermally-stratified air layers over solar-heated ground are exploited for scalable, low-cost power generation by the deliberate formation of intense buoyancy-induced vertical columnar vortices. Such vortices entrain the ground-heated air layer in regions with high surface solar heating rates, and convert the (gravitational) potential energy into "solar-induced wind" with significant kinetic energy. Unlike naturally occurring "dust devil" vortices, these columnar vortices are deliberately triggered and anchored. Each vortex is sustained by continuous entrainment of ground-heated air through an azimuthal array of stationary ground-mounted vertical vanes. Electric power is generated by using the rotational and vertical air motions within the columnar vortex to drive a vertical-axis turbine. Meter-scale laboratory experiments have demonstrated the nucleation, anchoring, and sustainment of strong, buoyancy-driven vortices centered within an azimuthal array of stationary vertical vanes above a controlled thermal ground plane. Outdoor tests of a meter-scale prototype have demonstrated autonomous start-up, formation, anchoring, and sustainment of a buoyancy-induced vortex generated solely by absorbed solar energy.
\end{abstract}

\section{Introduction}

In hot-climate regions, unstable, thermally-stratified atmospheric air layers heated from below by sun-warmed ground can result in spontaneous formation of buoyancyinduced, nominally axisymmetric, rising plumes. ${ }^{1}$ This buoyant stratification can evolve into a columnar atmospheric vortex, also known as a “dust devil,” by axial tilting of boundary layer vorticity concentrations generated within the vertically stratified, ground-heated air layer. ${ }^{2}$ These vortices convert the low-grade thermal energy available in the air layer over the warm surface into intense solar-induced air motions with significant angular and axial momenta. ${ }^{3}$ Field measurements of naturally occurring dust devil vortices by Sinclair $^{3}$ and Fitzjarrald ${ }^{4}$ demonstrated surface core diameters between 1 and 50 meters. Aerial measurements by Renno ${ }^{5}$ showed that large dust devils can extend as high as $1 \mathrm{~km}$. Such dust devils have considerable angular and axial momenta, with tangential and vertical wind speeds that can each exceed $50 \mathrm{~km} / \mathrm{hr}^{6}$ Stationary or "anchored" buoyancy-driven columnar vortices have been investigated in laboratory experiments using heated surfaces to mimic solar-heated ground. Unlike spontaneous dust devils in nature, deliberately triggered and anchored columnar vortices are stable and become self-sustained. Fitzjarrald ${ }^{7}$ used an azimuthal array of radial vanes to add angular momentum to the flow entrained by a central plume that formed over a heated plate. In a later study, Mullen and Maxworthy ${ }^{8}$ were able to measure temperature and tangential velocity distributions in the outer core flow, and elucidated the dependence of the vortex structure on surface heat flux.

Clean, renewable, electric power can be generated in hot climate or humid environments through exploitation of the thermal instability of the ground-heated air by deliberately triggering, anchoring, and sustaining arrays of stationary solar-driven vortices, each of which is coupled to a vertical-axis turbine. This power generation approach is simple, scalable, and low-cost. The present investigation focuses on the fundamental mechanisms of the formation, evolution, and dynamics of the columnar vortex, with particular emphasis on the direct coupling to a vertical-axis turbine in a $1 \mathrm{~m}$ scale laboratory facility. We also describe a similar outdoor prototype, powered only by solar radiation, with the vortex directly coupled to a vertical-axis turbine to demonstrate continuous rotation of the turbine with significant extraction of kinetic energy from the columnar vortex in the absence and presence of cross wind. A feasibility study of the power generation concept in a larger-scale outdoor prototype is planned within the next year.

\section{Power Generation Concept}

Nearly one-third of the global land mass is desert, providing huge untapped regions for capturing solar heat (about $200 \mathrm{~W} / \mathrm{m}^{2}$ averaged over a 24-hour day, and up to $1000 \mathrm{~W} / \mathrm{m}^{2}$ peak). The available power is competitive in magnitude with worldwide power generation from fossil sources. ${ }^{9}$ The essence of power generation by buoyancy- 
driven, anchored columnar vortices is to provide a new thermomechanical link between solar energy and electrical energy by harvesting vast amounts of low-grade solar heat for the sustainable production of electricity. Electric power is generated by deliberately forming these columnar vortices and coupling them to vertical-axis turbines, as shown schematically in Figure 1 . Unlike naturally occurring dust devil vortices that are free to wander laterally, and are hence susceptible to cross wind, each anchored columnar vortex is rendered stationary by an azimuthal array of tangential flow vanes. The rising air plume, at the center of this system of vanes, forces radial entrainment of ground-heated air along the surface. Passage through the stationary vanes imparts tangential momentum to the air flow. Once the vortex is formed, it is directly coupled to a vertical-axis wind turbine which extracts kinetic energy from the flow. The turbine can be specifically designed (and vertically placed) to maximize power extraction from the tangential and axial momenta of the vortex-induced flow.

The power that can be extracted from a single dust devil vortex can be estimated using a simple control volume analysis and data from field measurements of the axial and tangential velocity distributions within naturally-occurring vortices. Calculation of the power from the axial flow is similar to a typical horizontal axis wind turbine, ${ }^{2}$ while the power from the tangential flow for a turbine blade of a given geometry (characterized by the chord $c$ and span $R_{\mathrm{b}}$ ) is given by $P=2 \pi C_{\mathrm{p}} \rho \Omega^{3} R^{4} c\left[1+\ln \left(\mathrm{R}_{\mathrm{b}} \mathrm{R}\right)\right]$, where $C_{\mathrm{p}}$ is the turbine's Betz-limited coefficient of performance, $R$ is the vortex core radius, $\Omega$ is the angular velocity, and $\rho$ is the air density. Using the nominal tangential and axial wind speeds of $8 \mathrm{~m} / \mathrm{s}$ and $11 \mathrm{~m} / \mathrm{s}$, respectively, reported by Sinclair, ${ }^{6}$ a 5.2 diameter vortex, coupled to a $10.4 \mathrm{~m}$ diameter vertical-axis turbine, can deliver up to $50 \mathrm{kWe}$ to the grid, assuming an $80 \%$ generator efficiency and accounting for the Betz limit. For utility-scale applications, these $50 \mathrm{kWe}$ vortex units could be centered $55 \mathrm{~m}$ apart on a rectangular grid ( 320 units per $\mathrm{km}^{2}$, with $<4 \%$ of the total surface area within the swept area of the rotor) and have no significant mutual interference or depletion of the available thermal resources, based on a conservative vertical "make-up" air speed of $1 \mathrm{~m} / \mathrm{s}$ between turbine centers. Such an array of vortices has an estimated output of $16 \mathrm{MWe} / \mathrm{km}^{2}$, comparing favorably to conventional wind turbines (3-6 MWe $/ \mathrm{km}^{2}$ ) and solar photovoltaic/concentrated solar power (15-25 MWe/km²).

Using buoyancy-induced vortices for electric power generation has several key advantages compared to conventional wind turbine technology. Because the vortex harvests energy from the thin thermal layer nearest to the ground, the vertical-axis turbines are relatively close to the ground level, reducing operational and maintenance costs. This results in a much smaller system (stationary azimuthal flow vanes, turbine, generator, etc.) than conventional wind turbines, making the system cheaper (per peak We) to manufacture, transport, and install. Also, because the (gravitational) potential energy that the vortex uses is based on a temperature difference between the ground air layer and the air aloft, and the ground has a large thermal capacity, power output will experience much

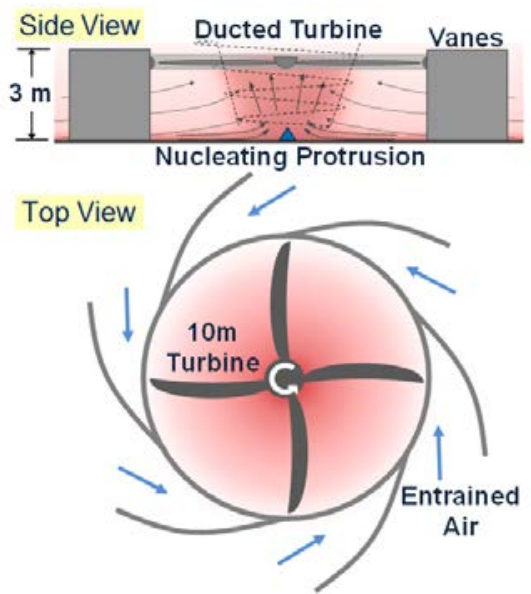

Figure 1 Deliberately triggered, stationary buoyancydriven vortex spinning a turbine: side and top views.

less intermittency than that from a conventional wind turbine. It is noteworthy that, in addition to the collection of the available gravitational potential energy in the solar-heated ground air layer, the azimuthal flow vanes can entrain considerable kinetic energy from ambient horizontal wind, independent of wind direction, thereby increasing the electric power generated.

Buoyancy-induced vortices also have several advantages compared to solar photovoltaic and concentrated solar power. First, the system requires no electromechanical control system to track the sun, rendering the system mechanically simpler and reducing initial capital and maintenance costs. Second, the vortex and turbine collect energy at a central location from a wide, unimproved area, so that the surface need not be tiled with expensive elements, again reducing the overall cost of the system. Finally, the thermal ground storage capability renders the electric power produced by buoyancy-induced vortices much less susceptible to cloud passage, and gives it much better diurnal predictability. The energy output of the vortex does not vanish immediately after sunset, and will, under some conditions, continue well into the evening. An estimate of the cost of energy (COE) completed by UTRC for the $50 \mathrm{kWe}(10 \mathrm{~m}$ diameter turbine) vortex unit found that the COE of such a system would be $\$ 0.066 / \mathrm{kWh}$. This figure is competitive with the current cost of energy for both solar PV $(\sim \$ 0.18 / \mathrm{kWh})$ and conventional wind ( $\$ 0.084 / \mathrm{kWh})$, based on the estimated lower operations and maintenance costs for the solar-induced vortex power generation technology.

\section{Laboratory Investigation of the Vortex}

The present investigation focuses on the controlled formation and characterization of a stationary (anchored) buoyancy-driven columnar vortex in a meter-scale test setup at Georgia Tech (Figures 2a and 2b). Ground heating is simulated by a planar $(1 \mathrm{~m} \times 1 \mathrm{~m})$, controllable heater placed on the underside of a $1 \mathrm{~m}^{2}$ aluminum plate used as the heated surface, where the power is adjusted based on thermocouple measurements of air temperature at several elevations and radii. Twelve thin adjustable 

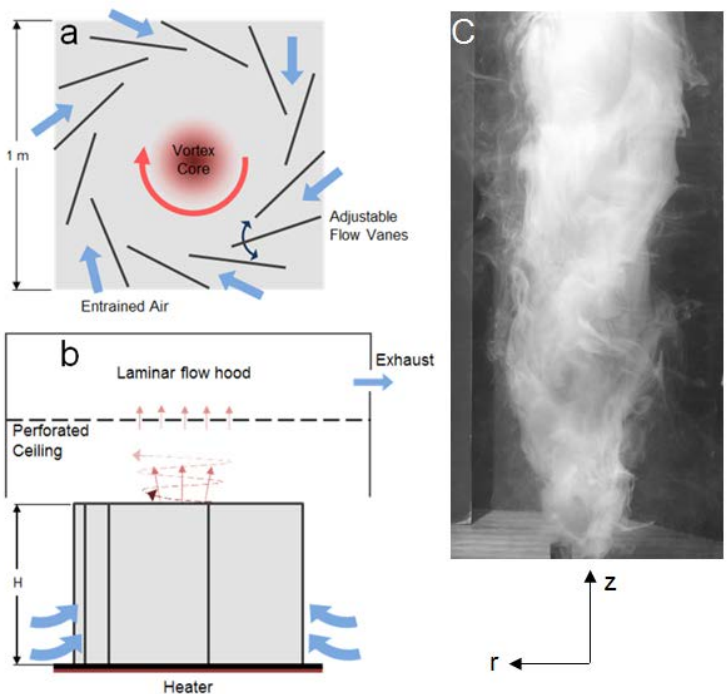

Figure 2 Buoyancy-induced columnar vortex: $a, b)$ top and side views of setup, and c) smoke visualization.

planar aluminum vanes $(40 \mathrm{~cm} \times 60 \mathrm{~cm})$ distributed azimuthally around the center of the plate impart tangential momentum to entrained makeup air, sustaining a central stationary buoyancy-driven vortex. The rising air above the heated plate is captured using an adjustable laminarflow hood (Figure 2b) with minimal disturbances to the vortex flow. The "ambient" temperature is controlled using the laboratory ventilation system. An array of sixteen thermocouples is used to measure radial temperature profiles in the vortex. In addition, the velocity field in the $r-\theta$ plane is measured using high-resolution stereo particle image velocimetry (PIV) using two $1600 \mathrm{x}$ 1200 pixel, 14-bit CCD cameras and an Nd:YAG laser. Smoke visualization of the self-sustained vortex core is shown in Figure 2c. The vortex generated in the laboratory can reach a height of about $4 \mathrm{~m}$, with a setupdependent surface core diameter of 5-25 cm.

The structure and scaling of a deliberately-triggered, buoyantly produced column vortex were investigated in the laboratory facility. Characteristic measurements of a "baseline" vortex structure were taken for a fixed stationary vane angle of $30^{\circ}$ (relative to the local radius) and surface temperature of $100^{\circ} \mathrm{C}$. The system is described in cylindrical coordinates $(r, \theta, z)$ with $z$ parallel to the vortex centerline, and the cylindrical components of the corresponding velocity field are given by $(u, v, w)$. Figures 3a shows the time-averaged radial distributions of the tangential, axial, and radial velocity components in the plane $z / R=0.18$ above the surface, where $R$ is the characteristic radius of the vortex where the tangential component has a maximum. These data show that tangential component dominates the flow, and the radial component is an order of magnitude smaller than the other components. The axial component is positive throughout the domain, reaching a maximum of $w=0.33 \Omega R$ near $r / R=1$. The temperature distribution in the $r-z$ plane within the "baseline" laboratory-scale column vortex is shown in Figure 3b. The measurements were performed from the edge of the heated surface $(r=0.5 \mathrm{~m})$ to the vortex centerline ( $r=0$ ) and up to $z=0.25 \mathrm{~m}$ above the surface using a thermocouple array (the location relative to a.

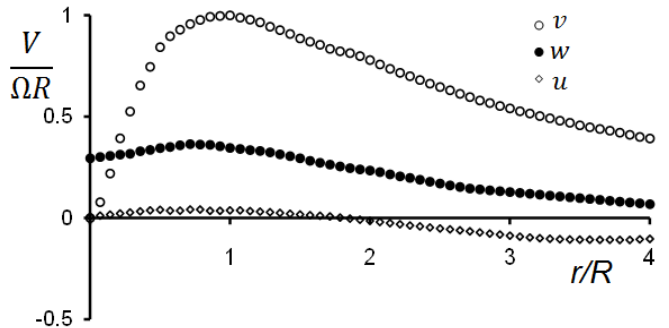

b.

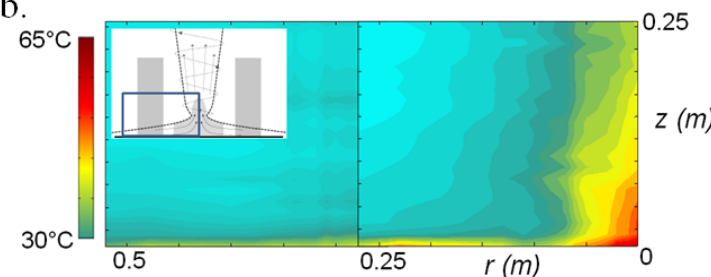

Figure 3 Radial distributions of tangential, axial, and radial velocity in the plane $\mathrm{z} / \mathrm{R}=0.18 \mathrm{~cm}(\mathrm{a})$, and temperature distribution in the $r$-z plane (b).

the column vortex is shown in the inset). The highest temperatures are measured within the core of the vortex, as all of the near-surface heated air is entrained directly into the core due to the low pressure generated by the rotating flow. Temperatures within the core are highest near the plate, and decrease away from the surface. At each elevation, the temperature is highest at the centerline, and quickly decreases to the ambient temperature at the edge of the vortex core.

To test the vortex-turbine interaction, a simple, $50 \mathrm{~cm}$ diameter slanted-blade, vertical-axis rotor (Figure 4a) was placed $30 \mathrm{~cm}$ above the center of the heated surface. The vortical flow below the rotor was measured using PIV, and radial distributions of the tangential velocity component in the absence and presence of the turbine are shown in Figure 4b. These data show that the presence of a spinning rotor driven by the flow (at $25 \mathrm{rpm}$ ) results

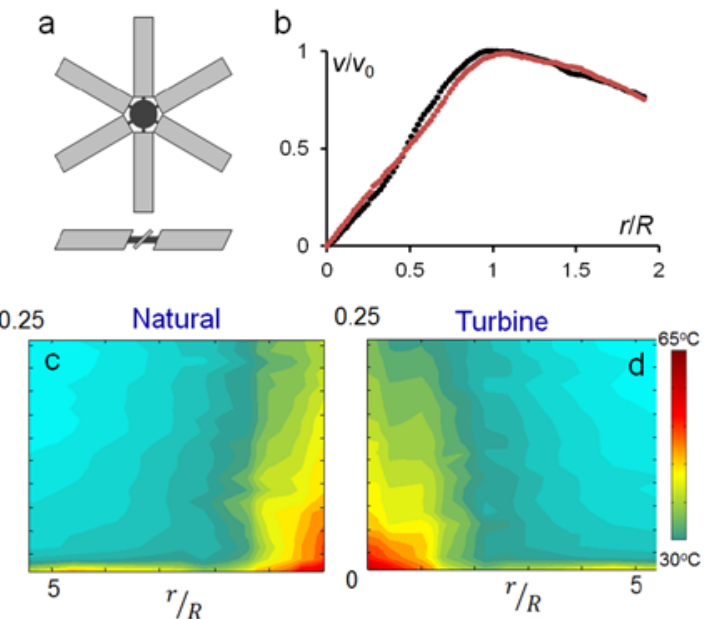

Figure 4 a) Top \& side views of simple, vertical-axis turbine (blade chord $7.5 \mathrm{~cm}$ span $23 \mathrm{~cm}$ ), and b) Radial profile of the tangential velocity component $(\mathrm{z}=0.6 \mathrm{R})$ in the "natural" vortex with (red) and without (black) the turbine. Comparison of the temperature distribution in the r-z plane for the vortex without (c) and with (d) the turbine present. 
RPM

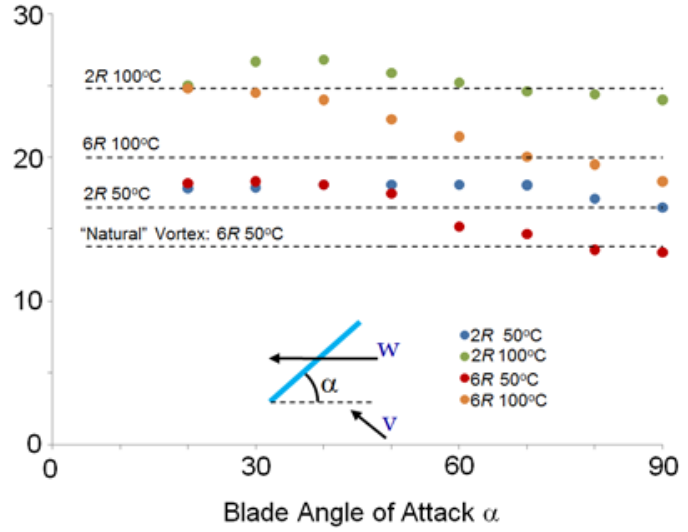

Figure 5 The angular speed of the vertical-axis rotor as a function of blade angle (with $90^{\circ}$ being parallel to the axial flow). Dashed lines represent the angular velocity of the vortex coupled to the rotor.

in negligible changes in the tangential velocity component of the vortex, with a reduction of about $3 \%$ in the maximum value. Corresponding measurements of temperature distributions within a planar cross section of the vortex core in the absence and presence of the rotor are shown in Figures 4c and 4d. In the presence of the rotor, the temperature distribution in the lower segment of the vortex is unchanged; however, spinning of the rotor mixes the core with the ambient air and cools the vortex core aloft.

The angular speed of the rotor was measured using the signal produced by directly coupling it to a simple AC generator. Angles of the blades are adjusted in order to affect the ratio of axial to angular momentum captured. Figure 5 shows the measured angular speed of the rotor versus blade angle for surface temperatures of $50^{\circ} \mathrm{C}$ and $100^{\circ} \mathrm{C}$ at $z=10$ and $30 \mathrm{~cm}(2 R$ and $6 R)$. The dashed lines in the figure represent the angular velocity of the vortex, calculated from the azimuthal velocity of the vortex core, which is undergoing approximately solid-body rotation. At $\alpha=90^{\circ}$, the blades are parallel to the axial flow, and thus capture only the rotational momentum from the flow. At this angle, the rotor spins at almost exactly the angular velocity of the columnar vortex. By decreasing the angle of the blades, additional energy is also captured from the axial flow, and the rotor spins at an increased speed. This approach thus allows for extraction of the power available from both the tangential and axial (vertical) flow.

The angular speed of the rotor was also measured for a range of temperature differences between the heated surface and the ambient (room temperature set at $21^{\circ} \mathrm{C}$ ). In addition to providing the approximate angular velocity of the vortex, the measured angular speed of the rotor can be used as a measure of the vortex strength, since the mechanical resistance of the generator remains nearly constant. The angular speed of the rotor was again measured using the signal from an AC generator, and the blades were set at $\alpha=45^{\circ}$ in order to capture both axial and angular momentum. The results are shown in Figure 6. It is seen that a minimum temperature difference of $5^{\circ} \mathrm{C}$ between the surface and the ambient is required to produce a columnar vortex with enough strength to drive the vertical-axis rotor. Thus, even on days where the solar

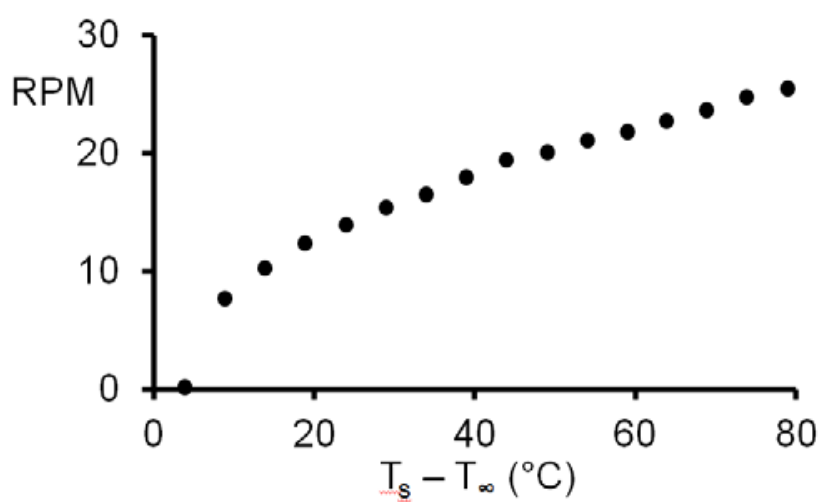

Figure 6 The angular speed of the vertical-axis turbine as a function of the temperature difference between the heated surface and the ambient.

radiation reaching the Earth's surface is significantly diminished, it might very well be possible to generate buoyancy-induced vortices with enough power to spin a turbine. In the laboratory, once the minimum temperature difference threshold is reached, the rate at which the turbine is spun increases monotonically with surface temperature. It is therefore desirable to produce a large temperature difference between the surface and the ambient air, which is achievable in an outdoor full-scale facility through use of materials that absorb a large fraction of the incident solar radiation.

\section{Outdoor Vortex Prototype}

A meter-scale outdoor prototype driven exclusively by solar radiation was constructed and tested at Georgia Tech (Figure 7). The prototype has twelve radiallylocated adjustable planar plywood flow vanes, each $40 \mathrm{~cm} \times 60 \mathrm{~cm}$. The vanes are located around a $1 \mathrm{~m}$ diameter, $2 \mathrm{~mm}$ thick steel plate painted black to increase absorption of solar radiation, which provided a surface temperature up to $30^{\circ} \mathrm{C}$ higher than the surrounding concrete surface. Testing of the outdoor prototype showed the creation and sustainment of a buoyancy-

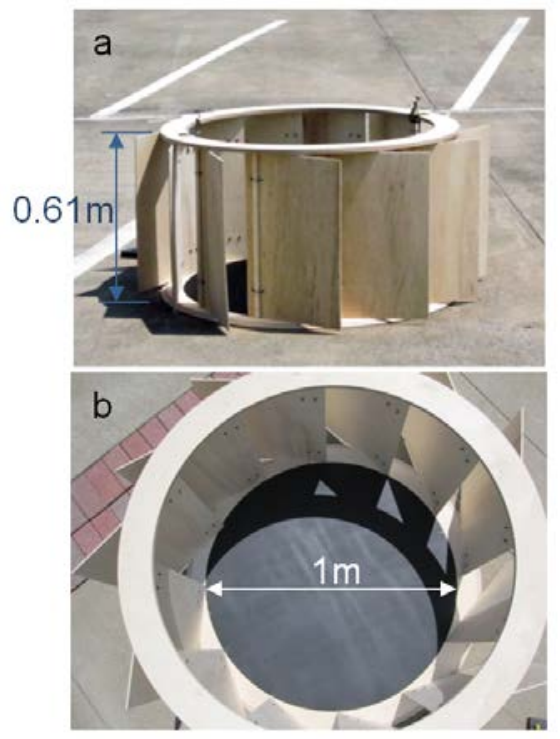

Figure 7 Meter-scale outdoor prototype of field facility: side (a) and top (b) view. 


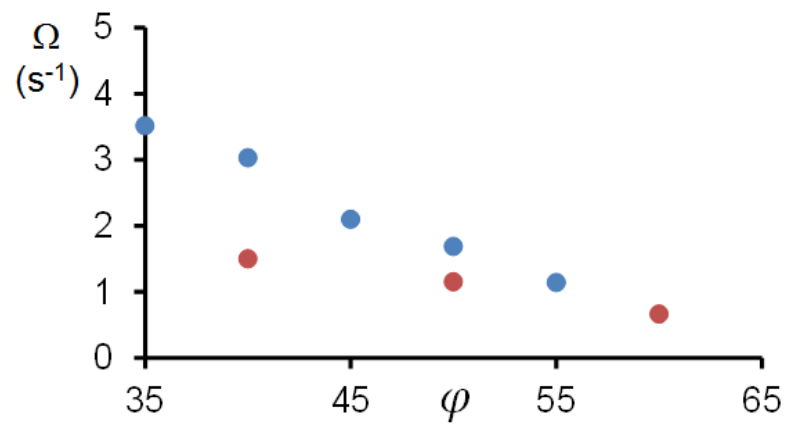

Figure 8 Variation of the vortex core angular velocity in the laboratory $(\bullet)$ and turbine in the outdoor $(\bullet)$ facility versus vane angle.

induced vortex on the scale of the laboratory vortex, generated solely by absorbed solar energy. The outdoor vortex was directly coupled to the simple vertical-axis rotor used in the laboratory, placed at $z=45 \mathrm{~cm}$, and achieved a turbine rotation rate of approximately $11 \mathrm{rpm}$, compared to $14 \mathrm{rpm}$ in the laboratory at the same temperature difference between the center of the plate and the ambient. The spinning of the rotor was also maintained in the presence and absence of cross wind.

The azimuthal flow vanes of the laboratory and outdoor facilities can be adjusted to affect the induced swirl of the entrained flow, altering the amount of rotational energy supplied to the vortex. The angular velocity of the laboratory vortex was measured for a series of flow vane angles at a similar $\Delta \mathrm{T}$, between the ambient and vortex core, as the vortex produced outdoors (Figure 8). The laboratory experiments showed that the core radius increases significantly with vane angle while the tangential velocity remains nominally constant, thereby leading to a decrease in the angular velocity of the columnar vortex. The effect of flow vane angle on the angular velocity of the turbine in the outdoor facility was also investigated and compared to results obtained in the laboratory facility (Figure 8). The rotational speed of the outdoor turbine is similar to the speed of the laboratory vortex for larger vane angles. As the vane angle is decreased, the smaller diameter of the vortex reduces the torque that the vortex core can apply to the turbine, and the difference between the angular velocities of the laboratory and outdoor turbines becomes larger. These results indicate that the vane angle can be adjusted to maximize the electric power output.

\section{Conclusions}

Power production from buoyancy-induced vortices provides a novel, low-cost, scalable method to harness vast amounts of low-grade thermal energy available in hotclimate regions for sustainable production of electricity. The essence of the power generation approach is to provide a link between solar energy and electrical energy by exploiting stratified, thermally-buoyant, solar-heated air layers that are heated from below by the sun-warmed ground. Deliberate triggering of the buoyant air leads to controlled formation of intense, stationary columnar vortices, sustained by continuous entrainment of solarheated air. Electric power is generated by using the angular and axial momentum of the vortex to drive a vertical-axis turbine coupled with an electric generator. Simple calculations show that for a vortex 5 meters in diameter with nominal tangential and axial wind speeds of $8 \mathrm{~m} / \mathrm{s}$ and $11 \mathrm{~m} / \mathrm{s}$ coupled to a $10 \mathrm{~m}$ diameter verticalaxis turbine, $50 \mathrm{kWe}$ could be extracted, in principle. An estimate for the cost of energy of the technology, $\$ 0.066$ per $\mathrm{kWh}$, is competitive with current renewable energy technologies.

Preliminary, meter-scale laboratory experiments at Georgia Tech have demonstrated formation and sustainment of a buoyancy-driven vortex that was investigated in some detail. The laboratory vortex was coupled with a simple, six-blade vertical-axis rotor which extracted significant amounts of kinetic energy and interfered minimally with formation of the vortex. Finally, sustainment of a buoyancy-driven vortex and operation of a rotor in an outdoor, meter-scale facility at Georgia Tech were successfully demonstrated in the absence and presence of a cross wind. It was shown that for the same temperature difference between the surface and the ambient, the laboratory and outdoor vortices can spin a turbine rotor at 14 and $11 \mathrm{rpm}$, respectively. The vortices produced outdoors were generated using only solar energy collected by the thermal ground plane, demonstrating proof-of-concept for converting low-grade heat available in the air layer overlying a warm surface into "concentrated wind" with significant kinetic energy.

Future investigation will focus on the construction of larger (2-3 m) outdoor prototype in the Arizona desert to further test the onset and sustainment of the columnar vortex and power extraction.

\section{References}

[1] Maxworthy, T. "A Vorticity Source for Large-Scale Dust Devils and Other Comments on Naturally Occurring Columnar Vortices,” J. Atm. Sci., 30, 1717-1722, 1973.

[2] Hau, E. "Wind Turbines: Fundamentals, Technologies, Application, Economics,” Springer, 2000.

[3] Sinclair, P.C. "General Characteristics of Dust Devils," $J$. App. Meteor., 8, 32-45, 1969.

[4] Fitzjarrald, D.E. “A Field Investigation of Dust Devils,” $J$. App. Met., 12, 808-813, 1973.

[5] Renno, N.O. "MATADOR 2002: A Pilot Field Experiment on Convective Plumes and Dust Devils," J. Geoph. Res. 109, E07001, 2004.

[6] Sinclair, P.C. "The Lower Structure of Dust Devils," J. Atm. Sci., 30, 1599-1619, 1973.

[7] Fitzjarrald, D.E., "A Laboratory Simulation of Convective Vortices,” J. Atm. Sci., 30 894-902, 1973.

[8] Mullen, J.B. and Maxworthy, T. "A Laboratory Model of Dust Devil Vortices,” Dyn. Atmos. Oceans, 1, 181-214, 1977.

[9] Meadows, D., Randers, J., and Meadows, D. “The Limits to Growth, The 30-Year Update," Chelsea Green Publishing Co., 2004. 